\title{
Synthesis of Nucleoside $\alpha$-Thiotriphosphates via an Oxathiaphospholane Approach
}

\author{
Konrad Misiura, Daria Szymanowicz, Wojciech J. Stec*
}

\section{SUPPORTING INFORMATION}

\section{General procedure for nucleoside 5'-O-(2-thio-1,3,2-oxathiaphospholanes) (2a-h):}

Protected nucleoside $(3 \mathrm{mmol})$ was dried by co-evaporation with dry pyridine $(2 \times 30 \mathrm{~mL})$ and re-dissolved in this solvent $(30 \mathrm{~mL})$. Into this solution sulfur $(0.48 \mathrm{~g}, 15 \mathrm{mmol})$ and 2-chloro-2-thio-1,3,2-oxathiaphospholane $(0.47 \mathrm{~g}, 3.3 \mathrm{mmol})$ were added and resulting mixture was left with stirring for $16 \mathrm{~h}$. Pyridine was evaporated and the residue was dissolved in toluene $(30 \mathrm{~mL})$ and evaporated again. Then the residue was dissolved in acetonitrile and precipitated solid was filter off. The filtrate was concentrated and obtained crude product was purified by means of silica-gel chromatography using chloroform supplemented with methanol (0-5\%) as an eluent. Appropriate fractions (TLC assay) were combined and concentrated to dryness to give product as a solid foam. The yields and physico-chemical characteristics of obtained $\mathbf{2 a - h}$ are presented in Table 1 and in Chart 1.

General procedure for nucleoside 5'-O-( $\boldsymbol{\alpha}$-thiotriphosphates) (1a-h): Oxathiaphospholane $\mathbf{2 a}-\mathbf{h}(0.3 \mathrm{mmol})$ was dissolved in dry $30 \mathrm{mM}$ solution of $\mathbf{3}^{1}(10 \mathrm{~mL})$ and resulting solution was additionally dry by keeping it with molecular sieves $3 \mathrm{~A}(0.6 \mathrm{~g})$ for $3 \mathrm{~h}$. Then DBU (50 $\mu \mathrm{L}, 0.33 \mathrm{mmol}$; stored over $\mathrm{CaH}_{2}$ ) was added. After $2 \mathrm{~h}$ reaction mixture was analyzed by ${ }^{31}$ P NMR (Fig. 1) and then concentrated and re-dissolved in a mixture of conc. ammonia (7.5 $\mathrm{mL})$ and acetonitrile $(1.5 \mathrm{~mL})$. For protected nucleoside $\alpha$-thiotriphosphate $5 \mathbf{e}$ deprotection took $2 \mathrm{~h}$ at room temperature; $6 \mathrm{~h}$ for $\mathbf{5 f}$ and $\mathbf{5 g}$, respectively). Complete removal of protecting groups from $\mathbf{5 a}, \mathbf{5 b}, \mathbf{5 c}, \mathbf{5 d}$, and $\mathbf{5 h}$ required heating for $6 \mathrm{~h}$ at bath temperature $55^{\circ} \mathrm{C}$. Obtained crude products were purified on DEAE -Sephadex A-25 (150 mL) using a linear gradient of TEAB buffer $\mathrm{pH} 7.5$ (3 L). Appropriate fractions were combined and concentrated to dryness to give desired product as a solid. Compounds $\mathbf{1 d}$ and $\mathbf{1 h}$ were additionally purified by RP-HPLC chromatography. The yields and physico-chemical characteristics of obtained 1a-h are presented in Table 2 and in Fig. 2-4.

\footnotetext{
${ }^{1}$ Tris(tetra- $n$-butylammonium pyrophosphate) obtained from sodium pyrophosphate and tetra- $n$-butylammonium hydroxide using Poulter's method (Davisson, V. J.; Woodside, A. B.; Neal, T. R.; Stremler, K. E.; Muehlbacher, M.; Poulter, C. D. J. Org. Chem. 1986, 51, 4768-4779). Acetonitrile solution of this compound was dried with molecular sieves $3 \mathrm{~A}(6-8 \mathrm{~h})$ until water content (cuolometric assay) was below $50 \mathrm{ppm}$.
} 


\section{Adenosine 5'-O-triphosphate (6)}

Solution of ATP $\alpha \mathrm{S}(\mathbf{1 f}, 0.1 \mathrm{mmol})$ in water $(1 \mathrm{~mL})$ was mixed with $0.1 \mathrm{M}$ solution of Oxone ${ }^{\circledR}$ buffered with sodium acetate until pH 7.0 was reached $(1.5 \mathrm{~mL}, 0.15 \mathrm{mmol})$, and left for $16 \mathrm{~h}$. Product was isolated by ion-exchange chromatography on DEAE-Sephadex A-25 $(50 \mathrm{~mL})$ using a linear gradient $(0.1-0.7 \mathrm{M})$ of TEAB buffer $\mathrm{pH}$ 7.5. Appropriate fractions were combined and concentrated to dryness to give desired product as a solid: $(0.063 \mathrm{mmol}$, $63 \%$ yield); $\mathrm{t}_{\mathrm{R}}(\mathrm{min})$ (conditions as above), 9.52; $\delta_{31 \mathrm{P}}\left(\mathrm{D}_{2} \mathrm{O}\right):-6,08(\mathrm{~d}, J 20.4 \mathrm{~Hz}),-11.15(\mathrm{~d}, J$ 19.4 Hz), -21.89 (m); MS-MALDI TOF m/z $506(\mathrm{M}-\mathrm{H})$.

In a similar way, using $1 \mathrm{M}$ solution of hydrogen peroxide $(1.5 \mathrm{~mL}, 1.5 \mathrm{mmol})$, compound 1f was converted into ATP with $65 \%$ yield.

\section{Thymidine 5'-O-( $\alpha$-thiodiphosphate) (7)}

Starting from $\mathbf{2 a}$ and using a general procedure as above, with replacement of $\mathbf{3}$ with bis(tetra- $n$-butylammonium) hydrogen phosphate ${ }^{2} \mathrm{dTDP} \alpha \mathrm{S}$ was obtained with $24 \%$ yield: $\mathrm{t}_{\mathrm{R}}$ (min) (conditions as above): 7.64, 8.02; $\delta_{31 \mathrm{P}}\left(\mathrm{D}_{2} \mathrm{O}\right): 41.58(\mathrm{~d}, J 30.1 \mathrm{~Hz}), 41.12(\mathrm{~d}, J 30.1 \mathrm{~Hz})$, $-8.34(\mathrm{~d}, J 30.1 \mathrm{~Hz})$; MS-MALDI TOF $m / z, 474(\mathrm{M}+\mathrm{Na})$.

\section{2',3'-O,O-Diacetyladenosine 5'-O-(2-seleno-1,3,2-oxathiaphospholane) (8)}

Starting from 2',3'-O, $\mathrm{O}$-diacetyladenosine and following a general procedure for nucleoside 5'-O-(2-thio-1,3,2-oxathiaphospholanes) elemental selenium was used instead of sulfur, and the title compound was obtained as a solid foam with $72 \%$ yield: TLC (chloroform/ethanol 9:1), $\mathrm{R}_{\mathrm{f}} 0.48 ; \delta_{1 \mathrm{H}}\left(\mathrm{CD}_{3} \mathrm{Cl}\right): 8.38(\mathrm{~s}, 1 \mathrm{H}),, 8.13(\mathrm{~s}, 1 \mathrm{H}), 6.30(\mathrm{~d}, J 6.3 \mathrm{~Hz}$, $1 \mathrm{H}), 5.75-5.89(\mathrm{~m}, 2 \mathrm{H}), 5.59-5.67(\mathrm{~m}, 1 \mathrm{H}), 4.34-4.65(\mathrm{~m}, 4 \mathrm{H}), 3.39-3.67$ (m, 2H), 2.16 (s, 3H), $2.06(\mathrm{~s}, 3 \mathrm{H}) ; \delta_{31 \mathrm{P}}\left(\mathrm{CD}_{3} \mathrm{Cl}\right): 100.00\left(J_{\mathrm{PSe}} 963 \mathrm{~Hz}\right), 99.93\left(J_{\mathrm{PSe}} 963 \mathrm{~Hz}\right) ; \mathrm{MS}-\mathrm{FAB}(+\mathrm{VE})$ $m / z 538.0(\mathrm{M}+\mathrm{H}, 1 \mathrm{Se})$.

\section{Adenosine 5'-O-( $\alpha$-selenotriphosphate) (9)}

Starting from 8 and using a general procedure as for preparation of nucleoside 5 ' $-O$ ( $\alpha$-thiotriphosphates), ATP $\alpha$ Se was obtained with $31 \%$ yield: $t_{R}(\min )$ (conditions as above): 9.19, 9.75; $\delta_{31 \mathrm{P}}\left(\mathrm{D}_{2} \mathrm{O}\right): 32.38\left(\mathrm{~d}, J_{\mathrm{PP}} 33.6, J_{\mathrm{PSe}} 790 \mathrm{~Hz}\right), 32.31\left(\mathrm{~d}, J_{\mathrm{PP}} 35.6, J_{\mathrm{PSe}} 791 \mathrm{~Hz}\right),-6.62$

\footnotetext{
${ }^{2}$ This compound was obtained from sodium dihydrogen phosphate and tetra- $n$-butylammonium hydroxide using the same procedure as described for $\mathbf{3}$ (see previous Note). Concentration of bis(tetra- $n$-butylammonium) hydrogen phosphate after treatment with molecular sieves was established by means ${ }^{31} \mathrm{P}$ NMR assay using weight amount of HMPT as an internal standard.
} 
(d, $J 21.1 \mathrm{~Hz}),-23.69(\mathrm{dd}, 33.6,21.1 \mathrm{~Hz}),-24.16(\mathrm{dd}, 35.6,21.1 \mathrm{~Hz}) ;$ MS-MALDI TOF $\mathrm{m} / z$ $576(\mathrm{M}, 1 \mathrm{Se})$.

\section{Adenosine 5'-O-( $\alpha$-selenodiphosphate) (10)}

Starting from $\mathbf{8}$ and using a general procedure as above, with an employment of bis(tetra- $n$-butylammonium) hydrogen phosphate, $\mathrm{ADP} \alpha \mathrm{Se}$ was obtained with $23 \%$ yield: $\mathrm{t}_{\mathrm{R}}$ (min) (conditions as above): 7.64, 8.02; $\delta_{31 \mathrm{P}}\left(\mathrm{D}_{2} \mathrm{O}\right): 29.92(\mathrm{~d}, J 37.1 \mathrm{~Hz}),-7.00(\mathrm{~d}, J 37.1 \mathrm{~Hz})$; MS-MALDI TOF $m / z 490$ (M, 1Se). 
Table 1. Yields and Physico-chemical characteristics of Oxathiaphospholanes 2a-h

\begin{tabular}{|c|c|c|c|c|c|}
\hline compound & $\begin{array}{l}\text { yield } \\
(\%) \\
\end{array}$ & $\begin{array}{c}\text { TLC }^{\mathrm{a}} \\
\mathrm{R}_{\mathrm{f}} \\
\end{array}$ & $\begin{array}{c}{ }^{31} \mathrm{P}-\mathrm{NMR}^{\mathrm{b}} \\
\delta(\mathrm{ppm}) \\
\end{array}$ & $\begin{array}{c}\text { FAB }(+\mathrm{VE}) \\
(\mathrm{M}+\mathrm{H}) \\
\end{array}$ & $\begin{array}{c}\text { FAB (-VE) } \\
(\mathrm{M}-\mathrm{H})\end{array}$ \\
\hline $2 \mathbf{a}$ & 80 & 0.48 & $\begin{array}{l}105.51 \\
105.27\end{array}$ & 423.2 & 421.3 \\
\hline $2 b$ & 84 & 0.51 & $\begin{array}{l}106.23 \\
106.08\end{array}$ & 536.0 & 533.9 \\
\hline $2 c$ & 79 & 0.55 & $\begin{array}{l}106.30 \\
106.12\end{array}$ & 511.9 & 510.1 \\
\hline $2 d$ & 81 & 0.38 & $\begin{array}{l}107.59 \\
107.51\end{array}$ & 518.3 & 516.3 \\
\hline $2 e$ & 75 & 0.45 & $\begin{array}{l}106.33 \\
106.15\end{array}$ & 467.2 & 465.2 \\
\hline $2 f$ & 80 & 0.38 & 106.48 & 490.2 & 488.1 \\
\hline $2 \mathrm{~g}$ & 78 & 0.41 & $\begin{array}{l}106.58 \\
106.51\end{array}$ & 508.1 & 506.1 \\
\hline $2 h$ & 73 & 0.43 & $\begin{array}{l}107.189 \\
107.044\end{array}$ & 610.1 & 608.1 \\
\hline
\end{tabular}

a developing system: chloroform/ethanol 9:1

${ }^{\mathrm{b}}$ spectra run in $\mathrm{CD}_{3} \mathrm{Cl}$ 


\section{Chart 1. ${ }^{1} \mathrm{H}$ NMR Spectra of Oxathiaphospholanes $\mathbf{2 a - h}$}

2a, $\quad{ }^{1} \mathrm{H}-\mathrm{NMR}\left(\mathrm{CDCl}_{3}\right) \delta 8.51(\mathrm{~d}, 1 \mathrm{H}, \mathrm{J}=7.3 \mathrm{~Hz}), 7.49(\mathrm{~d}, 1 \mathrm{H}, \mathrm{J}=20 \mathrm{~Hz}), 6.36-6.40(\mathrm{~m}, 1 \mathrm{H})$, $5.29(\mathrm{t}, 1 \mathrm{H}, \mathrm{J}=7.0 \mathrm{~Hz}), 4.32-4.59(\mathrm{~m}, 4 \mathrm{H}), 4.22(\mathrm{~s}, 1 \mathrm{H}), 3.49-3.69(\mathrm{~m}, 2 \mathrm{H}), 2.41-2.47$ $(\mathrm{m}, 1 \mathrm{H}), 2.13-2.20(\mathrm{~m}, 1 \mathrm{H}) ,2.11(\mathrm{~s}, 3 \mathrm{H}), 1.98(\mathrm{~s}, 3 \mathrm{H})$

2b, $\quad{ }^{1} \mathrm{H}-\mathrm{NMR}\left(\mathrm{CDCl}_{3}\right) \delta 9.13(\mathrm{~s}, 1 \mathrm{H}), 8.80(\mathrm{~s}, 1 \mathrm{H}), 8.36-8.40(\mathrm{~m}, 1 \mathrm{H}), 8.02(\mathrm{~d}, 2 \mathrm{H}, 7.6 \mathrm{~Hz})$, 7.60-7.61 (m, 1H), 7.53-7.54 (m, 2H), 6.53-6.60 (m, 1H), 5.47-5.52 (m, 1H), 4.33-4.56 (m, 5H), 3.46-3.54 (m, 2H), 2.85-2.93 (m, 1H), 2.67-2.72 (m, 1H), $2.15(\mathrm{~s}, 3 \mathrm{H})$

2c, $\quad{ }^{1} \mathrm{H}-\mathrm{NMR}\left(\mathrm{CDCl}_{3}\right) \delta 8.21-8.27(\mathrm{~m}, 1 \mathrm{H}), 7.89(\mathrm{~d}, 2 \mathrm{H}, \mathrm{J}=7.6 \mathrm{~Hz}), 7.50-7.62(\mathrm{~m}, 4 \mathrm{H})$, 6.30-6.36 (m, 1H), 5.26-5.32 (m, 1H), 4.34-4.55 (m, 5H), 3.48-3.59 (m, 2H), 2.78-2.84 (m, 1H), 2.10-2.16 (m, 4H), 1.64-2.09 (m, 1H)

2d, $\quad{ }^{1} \mathrm{H}-\mathrm{NMR}\left(\mathrm{CDCl}_{3}\right) \delta 12.07(\mathrm{~d}, 1 \mathrm{H}, \mathrm{J}=10.7 \mathrm{~Hz}), 8.73-8.84(\mathrm{~m}, 1 \mathrm{H}), 7.80(\mathrm{~d}, 1 \mathrm{H}$, $\mathrm{J}=3.1 \mathrm{~Hz}), 6.16-6.22(\mathrm{~m}, 1 \mathrm{H}), 5.51-5.57(\mathrm{~m}, 1 \mathrm{H}), 4.26-4.58(\mathrm{~m}, 5 \mathrm{H}), 3.43-3.55(\mathrm{~m}$, $2 \mathrm{H}), 3.09-3.26(\mathrm{~m}, 1 \mathrm{H}), 2.66-2.73(\mathrm{~m}, 1 \mathrm{H}), 2.43-2.51(\mathrm{~m}, 1 \mathrm{H}), 2.13(\mathrm{~s}, 3 \mathrm{H}), 1.27(\mathrm{~d}$, $6 \mathrm{H}, \mathrm{J}=6.8 \mathrm{~Hz})$

2e, $\quad{ }^{1} \mathrm{H}$ - NMR $\left(\mathrm{d}_{6}\right.$-DMSO) $\delta 11.45(\mathrm{~d}, 1 \mathrm{H}, \mathrm{J}=2 \mathrm{~Hz}), 7.64-7.70(\mathrm{~m}, 1 \mathrm{H}), 5.88-5.91(\mathrm{~m}, 1 \mathrm{H})$, 5.66-5.71 (m, 1H), 5.26-5.42 (m, 2H), 4.62-4.30 (m, 2H), 4.27-4.20 (m, 2H), 3.52-3.75 (m, 2H), $2.06(\mathrm{~s}, 1 \mathrm{H}), 2.05(\mathrm{~s}, 3 \mathrm{H}), 2.01(\mathrm{~s}, 3 \mathrm{H})$,

2f, $\quad{ }^{1} \mathrm{H}-: \mathrm{NMR}\left(\mathrm{d}_{6}-\mathrm{DMSO}\right) \delta 8.28(\mathrm{~s}, 1 \mathrm{H}), 8.14(\mathrm{~s}, 1 \mathrm{H}), 7.36-7.40(\mathrm{~m}, 2 \mathrm{H}), 6.18(\mathrm{~d}, 1 \mathrm{H}$, $\mathrm{J}=5.6 \mathrm{~Hz}), 5.93-6.00(\mathrm{~m}, 1 \mathrm{H}), 5.56-5.61(\mathrm{~m}, 1 \mathrm{H}), 4.23-4.57(\mathrm{~m}, 4 \mathrm{H}), 3.46-3.66(\mathrm{~m}$, 2H), 2.56-2.81 (m, 1H), $2.09(\mathrm{~s}, 3 \mathrm{H}), 2.05(\mathrm{~s}, 3 \mathrm{H})$,

2g, $\quad{ }^{1} \mathrm{H}-\mathrm{NMR}\left(\mathrm{d}_{6}\right.$-DMSO) $\delta 10.99(\mathrm{~s}, 1 \mathrm{H}), 8.10(\mathrm{~d}, 1 \mathrm{H}, \mathrm{J}=7.4 \mathrm{~Hz}), 7.22(\mathrm{~d}, 1 \mathrm{H}, \mathrm{J}=7.5 \mathrm{~Hz})$, 5.92-6.83 (m, 1H), 5.47-5.50 (m, 1H), 5.36-5.39 (m, 1H), 4.53-4.60 (m, 1H), 4.33-4.43 (m, 3H), 4.24-4.32 (m, 1H), 3.61-3.74 (m, 2H), $2.11(\mathrm{~s}, 3 \mathrm{H}), 2.08(\mathrm{~s}, 3 \mathrm{H}), 2.07(\mathrm{~s}, 3 \mathrm{H})$

2h, $\quad{ }^{1} \mathrm{H}-\mathrm{NMR}\left(\mathrm{CDCl}_{3}\right) \delta 12.22(\mathrm{~d}, 1 \mathrm{H}, \mathrm{J}=22 \mathrm{~Hz}), 9.28(\mathrm{~d}, 1 \mathrm{H}, \mathrm{J}=2.4 \mathrm{~Hz}), 8.06(\mathrm{dd}, 2 \mathrm{H}$, $\mathrm{J}=7.6 \mathrm{~Hz}, \mathrm{~J}=2.4 \mathrm{~Hz}), 7.78(\mathrm{~d}, 1 \mathrm{H}, \mathrm{J}=5.2 \mathrm{~Hz}), 7.64-7.67$ (m, 1H), 7.53-7.57 (m, 2H), 6.16$6.18(\mathrm{~m}, 1 \mathrm{H}), 5.95-6.08(\mathrm{~m}, 1 \mathrm{H}), 4.20-4.43(\mathrm{~m}, 6 \mathrm{H}), 3.42-3.47(\mathrm{~m}, 1 \mathrm{H}), 3.33-3.38(\mathrm{~m}$, $1 \mathrm{H}), 2.12-2.30(\mathrm{~m}, 6 \mathrm{H})$ 
Table 2. The Yields and Physico-chemical Characteristics of Nucleoside $\alpha$-Thiotriphoshates 1a-h

\begin{tabular}{|c|c|c|c|c|c|}
\hline compound & $\begin{array}{c}\mathrm{t}_{\mathrm{R}}^{\mathrm{a}} \\
\text { (min.) }\end{array}$ & $\begin{array}{c}\text { MS } \\
\text { MALDI }\end{array}$ & $\begin{array}{c}{ }^{31} \mathrm{P}-\mathrm{NMR}{ }^{\mathrm{b}} \\
\delta(\mathrm{ppm})\end{array}$ & $\begin{array}{r}\mathrm{J}_{\mathrm{P} \alpha-\mathrm{P} \beta} \\
(\mathrm{Hz})\end{array}$ & $\begin{array}{r}J_{\mathrm{P} \beta-\mathrm{P} \gamma} \\
(\mathrm{Hz}) \\
\end{array}$ \\
\hline $1 \mathbf{a}$ & 7.16 & \multirow{2}{*}{497} & SP 44.07(d), -23.07 (m), -9.51(d) & 32.4 & 17.7 \\
\hline$(\mathrm{dTTP} \alpha \mathrm{S})$ & 7.55 & & RP 43.68(d), $-23.07(m),-9.51(\mathrm{~d})$ & 30.0 & 17.7 \\
\hline $\mathbf{1 b}$ & 8.07 & \multirow{2}{*}{506} & SP 45.08(d), -20.53(m), -5.03(d) & 26.7 & 17.0 \\
\hline$(\mathrm{dATP} \alpha \mathrm{S})$ & 8.30 & & $\mathrm{RP}_{\mathrm{P}} 44.75(\mathrm{~d}),-20.65(\mathrm{~m}),-5.03(\mathrm{~d})$ & 25.9 & 17.0 \\
\hline 1c & 5.85 & \multirow{2}{*}{482} & $\mathrm{SP} 44.37(\mathrm{~d}),-22.14(\mathrm{~m}),-7.80(\mathrm{~d})$ & 26.5 & 18.4 \\
\hline$(\mathrm{dCTP} \alpha \mathrm{S})$ & 6.23 & & $\mathrm{RP}_{\mathrm{P}} 44.05(\mathrm{~d}),-22.42(\mathrm{~m}),-7.80(\mathrm{~d})$ & 26.6 & 18.4 \\
\hline 1d & 7.20 & \multirow{2}{*}{522} & SP 44.78(d), -21.53(m), -7.55(d) & 24.1 & 16.4 \\
\hline$(\mathrm{dGTP} \alpha \mathrm{S})$ & 7.39 & & $\mathrm{RP}_{\mathrm{P}} 44.52(\mathrm{~d}),-21.80(\mathrm{~m}),-7.55(\mathrm{~d})$ & 24.8 & 16.4 \\
\hline 1e & 7.36 & \multirow{2}{*}{499} & $\mathrm{SP} 43.29(\mathrm{~d}),-23.17(\mathrm{~m}),-8.44(\mathrm{~d})$ & 27.4 & 19.6 \\
\hline$(\mathrm{UTP} \alpha \mathrm{S})$ & 7.82 & & $\mathrm{RP}_{\mathrm{P}} 42.95(\mathrm{~d}),-23.64(\mathrm{~m}),-8.44(\mathrm{~d})$ & 27.8 & 19.6 \\
\hline 1f & 8.02 & \multirow{2}{*}{522} & $\mathrm{SP}_{\mathrm{P}} 43.67(\mathrm{~d}),-23.80(\mathrm{~m}),-10.56(\mathrm{~d})$ & 27.2 & 19.7 \\
\hline$(\mathrm{ATP} \alpha \mathrm{S})$ & 8.45 & & $\mathrm{RP}_{\mathrm{P}} 43.32(\mathrm{~d}),-24.25(\mathrm{~m}),-10.56(\mathrm{~d})$ & 27.9 & 19.7 \\
\hline $1 g$ & 7.35 & \multirow{2}{*}{498} & $S_{P} 43.60(d),-23.65(m),-10.39(d)$ & 27.5 & 19.5 \\
\hline$(\mathrm{CTP} \alpha \mathrm{S})$ & 7.75 & & $\mathrm{RP}_{4} 4.25(\mathrm{~d}),-24.07(\mathrm{~m}),-10.39(\mathrm{~d})$ & 27.9 & 20.4 \\
\hline 1h & 6.95 & \multirow{2}{*}{538} & SP 43.77(d), -22.08(m), -5,24(d) & 31.1 & 20.4 \\
\hline$(\mathrm{GTP} \alpha \mathrm{S})$ & 7.40 & & $\mathrm{RP}_{\mathrm{P}} 43.48(\mathrm{~d}),-22.20(\mathrm{~m}),-5,24(\mathrm{~d})$ & 29.8 & 20.4 \\
\hline
\end{tabular}

${ }^{\mathrm{a}}$ HPLC in $0.1 \mathrm{M}$ TEAB, pH 7.5 with $0 \rightarrow 60 \%$ acetonitrile in 20 min.; ${ }^{\mathrm{b}}$ spectra run in $\mathrm{D} 2 \mathrm{O}$ 
Fig. $1 .{ }^{31} \mathrm{P}$ NMR Spectra of Reaction Mixture of Oxathiaphospholane 2a with "wet" pyrophosphate $\mathbf{3}$ in a presence of DBU.

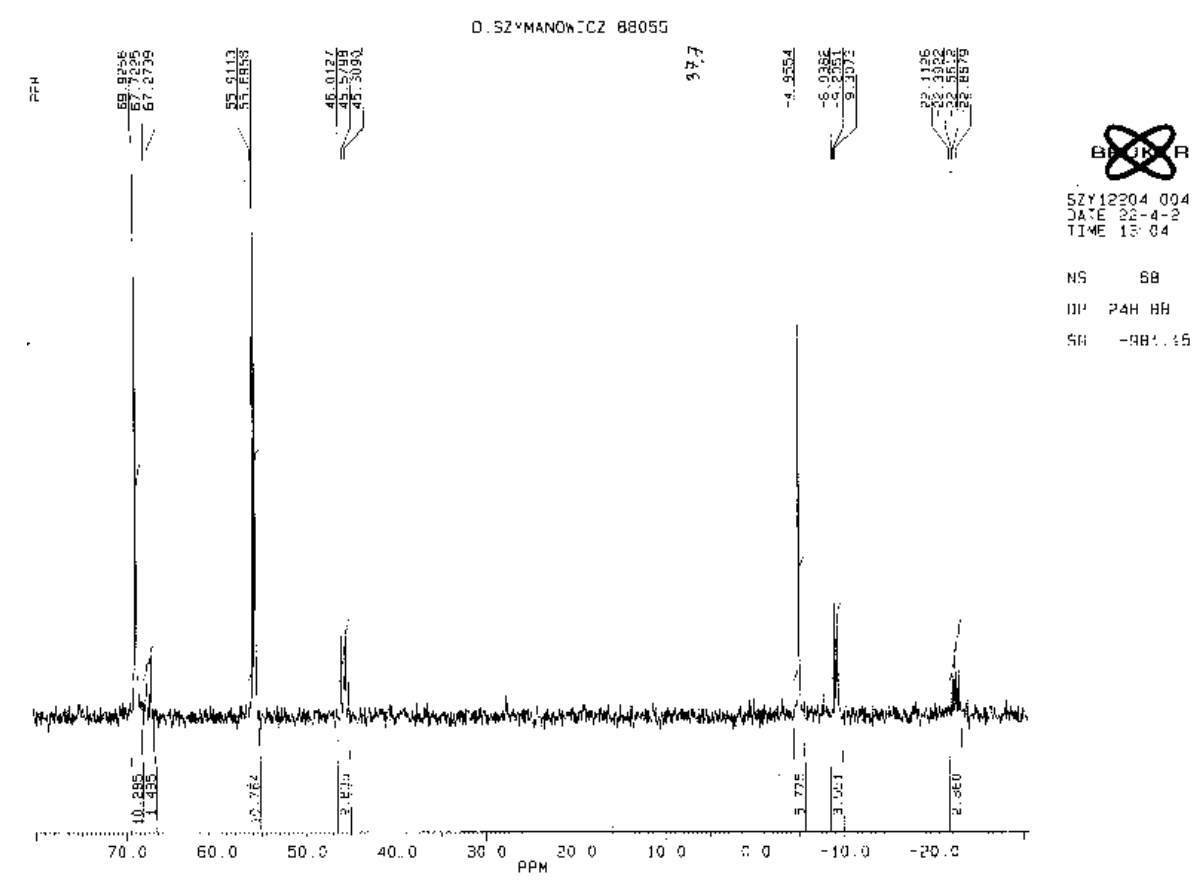


Fig. 2. HPLC Trace of $\mathbf{1 f}(\mathrm{ATP} \alpha \mathrm{S})$

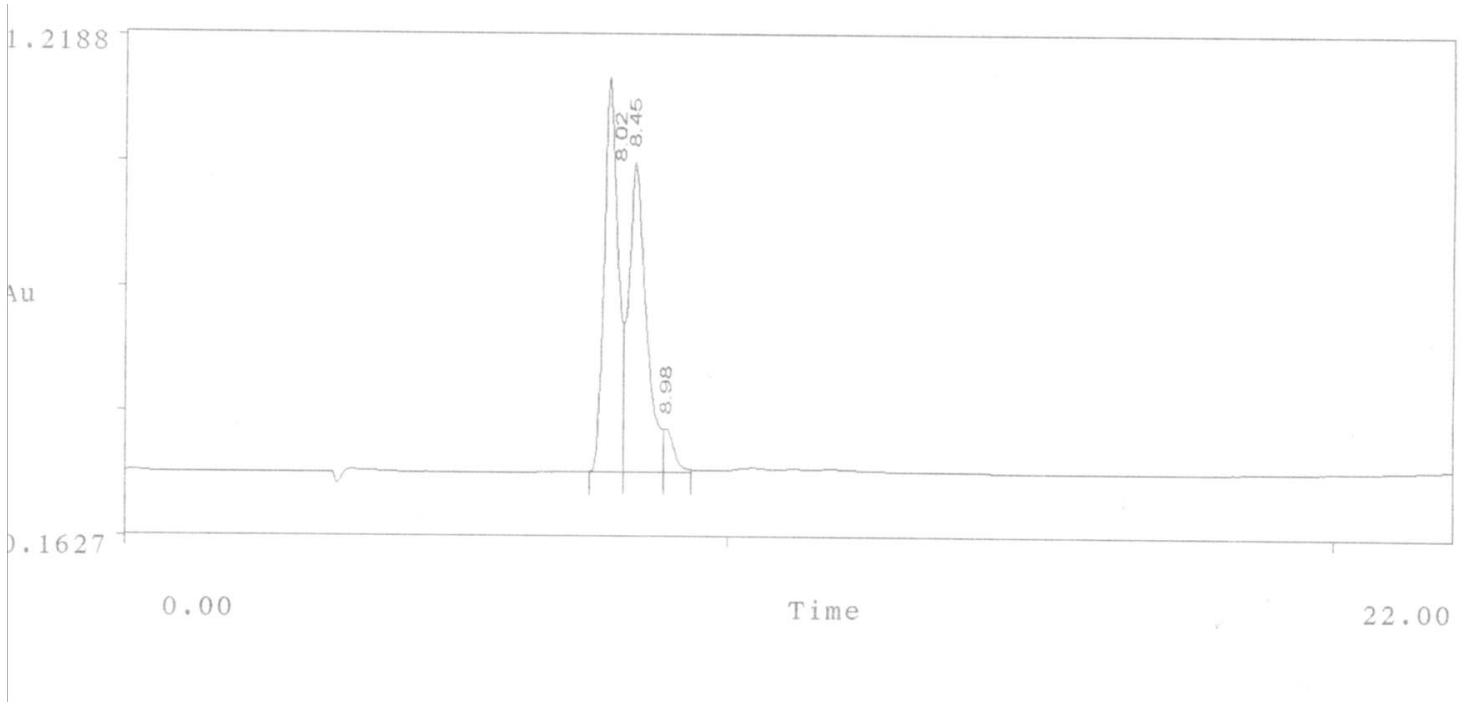


Fig. 3. ${ }^{31}$ P NMR Spectra of $\mathbf{1 f}(\mathrm{ATP} \alpha \mathrm{S})$

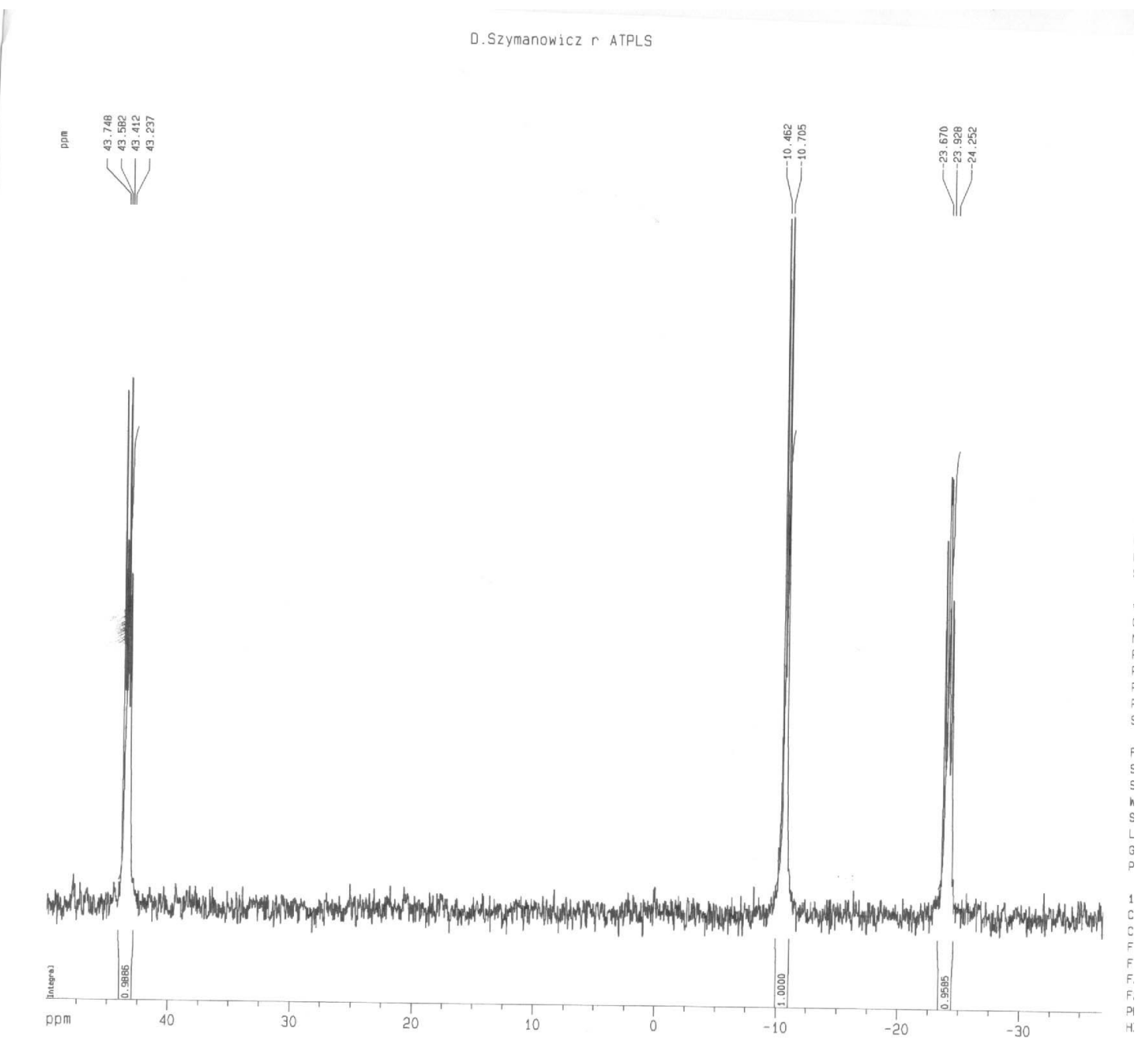


Fig. 4. MALDI-TOF MS Spectra of $\mathbf{1 f}(\mathrm{ATP} \alpha \mathrm{S})$

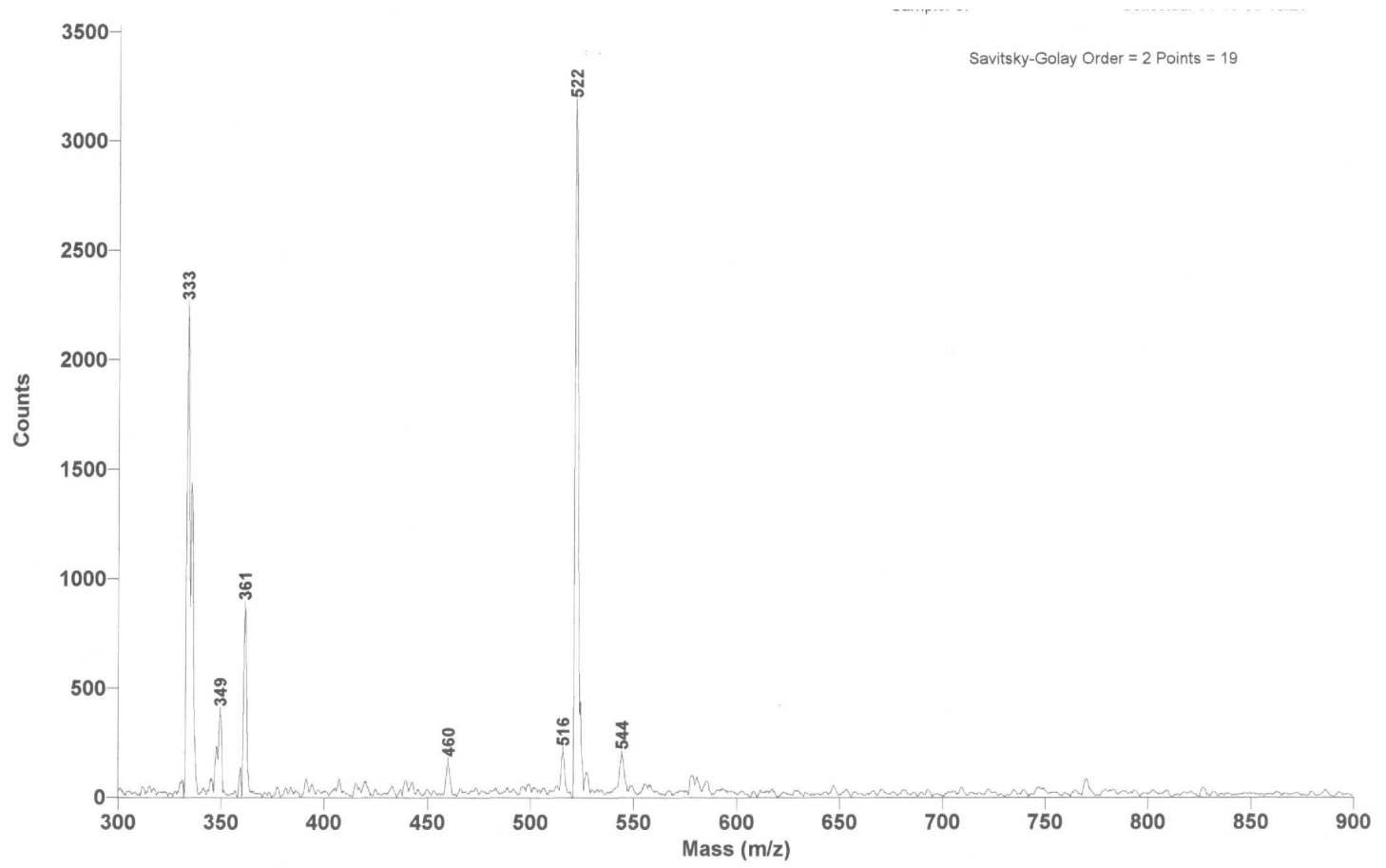


Fig. 5. MALDI-TOF MS Spectra of "dimer" obtained from 4 by ammoniacal deacetylation.

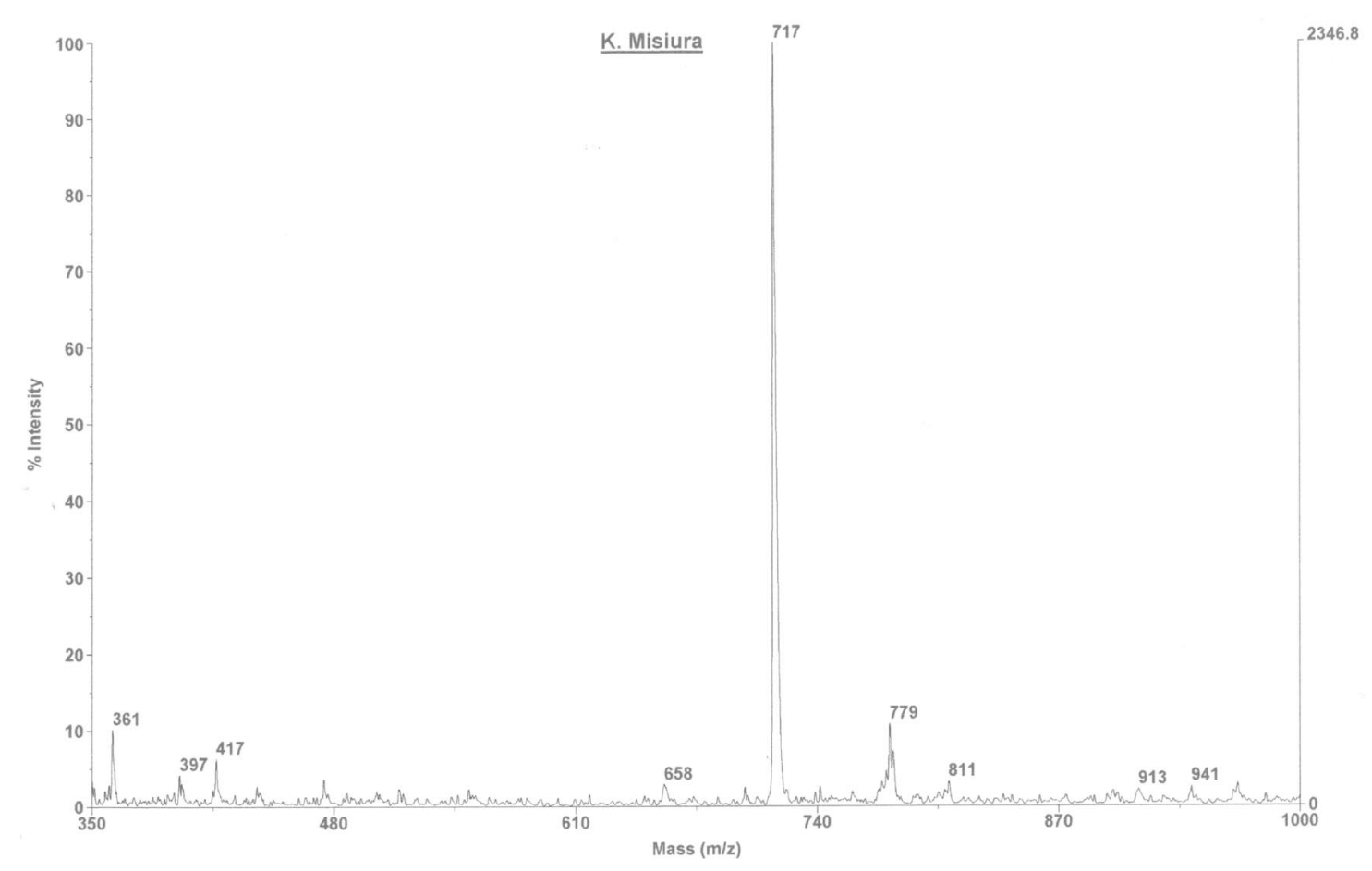

